\title{
Extremal numbers for odd cycles
}

\author{
Zoltan Füredi` and David S. Gunderson ${ }^{\dagger}$ \\ * Rényi Institute of Mathematics, Hungarian Academy of Sciences \\ E-mail: z-furedi@illinois.edu, furedi.zoltan@renyi.mta.hu \\ $\dagger$ University of Manitoba, Winnipeg, Canada \\ E-mail: David.Gunderson@umanitoba.ca
}

\begin{abstract}
We describe the $C_{2 k+1}$-free graphs on $n$ vertices with maximum number of edges. The extremal graphs are unique for $n \notin\{3 k-1,3 k, 4 k-2,4 k-1\}$. The value of ex $\left(n, C_{2 k+1}\right)$ can be read out from the works of Bondy [3], Woodall [14, and Bollobás [1, but here we give a new streamlined proof. The complete determination of the extremal graphs is also new.

We obtain that the bound for $n_{0}\left(C_{2 k+1}\right)$ is $4 k$ in the classical theorem of Simonovits, from which the unique extremal graph is the bipartite Turán graph.
\end{abstract}

* Research supported in part by the Hungarian National Science Foundation OTKA 104343, and by the European Research Council Advanced Investigators Grant 267195.

$\dagger$ Research supported by NSERC Discovery grant 228064.

MSC-class: 05C35, 05D99 Furedi'Gunderson exoddcycles $20133^{*} 10^{`} 24 \quad 24$ October 2013

Keywords: Turan graph problem, extremal graphs, odd cycles Printed on October 28, 2013 


\section{Introduction, exact Turán numbers}

Given a class of simple graphs $\mathcal{F}$ let us call a graph $\mathcal{F}$-free if it contains no copy of $F$ as a (not necessarily induced) subgraph for each $F \in \mathcal{F}$. Let $\operatorname{ex}(n ; \mathcal{F})$ denote the maximal number of edges in an $\mathcal{F}$-free graph on $n$ vertices. If the class of graphs $\mathcal{F}=$ $\left\{F_{1}, F_{2}, \ldots\right\}$ consists of a single graph then we write ex $(n ; F)$ instead of $\operatorname{ex}(n ;\{F\})$.

Let $T_{n, p}$ denote the Turán graph, the complete equi-partite graph, $K_{n_{1}, n_{2}, \ldots, n_{p}}$ where $\sum_{i} n_{i}=n$ and $\lfloor n / p\rfloor \leq n_{i} \leq\lceil n / p\rceil$. By Turán's theorem [12, 13] we have $\operatorname{ex}\left(n ; K_{p+1}\right)=e\left(T_{n, p}\right)$; furthermore, $T_{n, p}$ is the unique $K_{p+1}$-free graph that attains the extremal number. The case $\operatorname{ex}\left(n ; K_{3}\right)=\left\lfloor n^{2} / 4\right\rfloor$ was shown earlier by Mantel [10].

There are very few cases when the Turán number $\operatorname{ex}(n ; \mathcal{F})$ is known exactly for all $n$. One can mention the case when $F=M_{\nu+1}$ is a matching of a given size, $\nu+1$. Erdős and Gallai [6] showed that

$$
\operatorname{ex}\left(n, M_{\nu+1}\right)=\max \left\{\left(\begin{array}{c}
2 \nu+1 \\
2
\end{array}\right),\left(\begin{array}{l}
\nu \\
2
\end{array}\right)+\nu(n-\nu)\right\} .
$$

For the path of $k$ vertices Erdös and Gallai [6] proved an asymptotic and $\operatorname{ex}\left(n ; P_{k}\right)$ was determined for all $n$ and $k$ by Faudree and Schelp [7] and independently by Kopylov [9]. Erdös and Gallai [6] proved an asymptotic for the class of long cycles $\mathcal{C}_{\geq \ell}:=\left\{C_{\ell}, C_{\ell+1}, C_{\ell+2}, \ldots\right\}$. The exact value of the Turán number $\operatorname{ex}\left(n ; \mathcal{C}_{\geq \ell}\right)$ was determined by Woodall [15] and independently and at the same time by Kopylov [9].

There is one outstanding result which gives infinitely many exact Turán numbers, Simonovits' chromatic critical edge theorem [11]. It states that if $\min \{\chi(F): F \in$ $\mathcal{F}\}=p+1 \geq 3$ and there exists an $F \in \mathcal{F}$ with an edge $e \in E(F)$ such that by removing this edge one has $\chi(F-e) \leq p$, then there exists an $n_{0}(\mathcal{F})$ such that $T_{n, p}$ is the only extremal graph for $\mathcal{F}$ for $n \geq n_{0}$. The authors are not aware of any (nontrivial) further result when $\operatorname{ex}(n, \mathcal{F})$ is known for all $n$, neither any $F$ for which the value of $n_{0}(F)$ had been determined, except the case of odd cycle discussed below.

\section{The result, the extremal graphs without $C_{2 k+1}$}

The aim of this paper is to determine the Turán number of odd cycles for all $n$ and $C_{2 k+1}$ together with the extremal graphs. The value of $\operatorname{ex}\left(n, C_{2 k+1}\right)$ can be read out from the works of Bondy [2, 3], Woodall [14], and Bollobás [1] (pp. 147-156) concerning (weakly) pancyclic graphs. For a recent presentation see Dzido [5] who also considered the Turán number of wheels. But here we give a new streamlined proof and a complete description of the extremal graphs. 
Since $K_{\lceil n / 2\rceil,\lfloor n / 2\rfloor}$ contains no odd cycles, for any $k \geq 1$, ex $\left(n ; C_{2 k+1}\right) \geq\left\lfloor n^{2} / 4\right\rfloor$. For $C_{3}$ here equality holds for all $n$ with the only extremal graph is $T_{n, 2}$ by the Turán-Mantel's theorem. From now on, we suppose that $2 k+1 \geq 5$. Also for $n \leq 2 k$ obviously ex $\left(n, C_{2 k+1}\right)=\left(\begin{array}{l}n \\ 2\end{array}\right)$ so we may suppose that $n \geq 2 k+1$.

Every edge of an odd cycle is color critical so Simonovits' theorem implies that the complete bipartite graph is the only extremal graph and $\operatorname{ex}\left(n ; C_{2 k+1}\right)=e\left(T_{n, 2}\right)=$ $\left\lfloor n^{2} / 4\right\rfloor$ for $n \geq n_{0}\left(C_{2 k+1}\right)$. After choosing the right tools we present a streamlined proof and show that $n_{0}\left(C_{2 k+1}\right)=4 k$ (in case of $2 k+1 \geq 5$ ).

We define two classes of $C_{2 k+1}$-free graphs which could have at least as many edges as $T_{n, 2}$ for $n \leq 4 k-1$. A cactus $B\left(n ; n_{1}, \ldots, n_{s}\right.$ ) (for $n \geq 2, s \geq 1$ with $\left.\sum_{i}\left(n_{i}-1\right)=n-1\right)$ is a connected graph where the 2-connected blocks are complete graphs of sizes $n_{1}, \ldots, n_{s}$. Let us denote by $g(n, k)$ the largest size of an $n$-vertex cactus avoiding $C_{2 k+1}$. For this maximum all block sizes should be exactly $2 k$ but at most one which is smaller. Write $n$ in the form $n=(s-1)(2 k-1)+r$ where $s \geq 1$, $2 \leq r \leq 2 k$ are integers. Then

$$
g(n, k)=(s-1)\left(\begin{array}{c}
2 k \\
2
\end{array}\right)+\left(\begin{array}{l}
r \\
2
\end{array}\right) .
$$

Note that $g(n, k)>\left\lfloor n^{2} / 4\right\rfloor$ for $3 \leq n \leq 4 k-3$ and we have $g(n, k)=e\left(T_{n, 2}\right)=\left\lfloor n^{2} / 4\right\rfloor$ if $n \in\{4 k-2,4 k-1\}$. Thus the Simonovits threshold $n_{0}\left(C_{2 k+1}\right)$ is at least $4 k$.

For $n \geq k$, define the graph $H_{1}(n, k)$ on $n$ vertices by its degree sequence; it has $k$ vertices of degree $n-1$ and all other vertices have degree $k$. Then $H_{1}(n, k)$ is a complete bipartite graph $K_{k, n-k}$, together with all possible edges added in the first partite set. This graph does not contain the cycle $C_{2 k+1}$. Letting $h_{1}(n, k)$ denote the size of $H_{1}(n, k)$,

$$
h_{1}(n, k)=\left(\begin{array}{l}
k \\
2
\end{array}\right)+k(n-k) .
$$

Note that $h_{1}(n, k) \leq g(n, k)$ for all $k \leq n$ and here equality holds if $n$ is in the form $n=(s-1)(2 k-1)+r$ where $s \geq 1$ and $r \in\{k, k+1\}$.

Theorem 1. For any $n \geq 1$ and $2 k+1 \geq 5$,

$$
\operatorname{ex}\left(n ; C_{2 k+1}\right)= \begin{cases}\left(\begin{array}{l}
n \\
2
\end{array}\right) & \text { for } n \leq 2 k \\
g(n, k) & \text { for } 2 k+1 \leq n \leq 4 k-1 \text { and } \\
\left\lfloor n^{2} / 4\right\rfloor & \text { for } n \geq 4 k-2 .\end{cases}
$$

Furthermore, the only extremal graphs are $K_{n}$ for $n \leq 2 k ; B(n ; 2 k, n-2 k+1)$ for $2 k+1 \leq n \leq 4 k-1 ; H_{1}(n, k)$ for $n \in\{3 k-1,3 k\}$; and the complete bipartite graph $K_{\lceil n / 2\rceil,\lfloor n / 2\rfloor}$ for $n \geq 4 k-2$. 
Füredi-Gunderson: Extremal numbers for odd cycles

\section{A lemma on 2-connected graphs without $C_{2 k+1}$}

Lemma 2. Suppose that $n \geq 2 k+1 \geq 5$ and $G$ is a 2 -connected, $C_{2 k+1}$-free, nonbipartite graph with at least $\left\lfloor n^{2} / 4\right\rfloor$ edges. Then $e(G) \leq \operatorname{ex}\left(n ; C_{2 k+1}\right)$ and here equality holds only if $n \in\{3 k-1,3 k\}$ and $G=H_{1}(n, k)$.

For $5 \leq 2 k+1 \leq n$, define the graph $H_{2}(n, k)$ on $n$ vertices and

$$
h_{2}(n, k):=\left(\begin{array}{c}
2 k-1 \\
2
\end{array}\right)+2(n-2 k+1)
$$

edges, consisting of a complete graph $K_{2 k-1}$ containing two special vertices which are connected to all other vertices. Then $H_{2}(n, k)$ is a 2-connected $C_{2 k+1}$-free graph. For $k=2$ the graphs $H_{1}(n, k)$ and $H_{2}(n, k)$ are isomorphic. Recall a result of Kopylov [9] in a form we use it: Suppose that the 2-connected graph $G$ on $n$ vertices contains no cycles of length $2 k+1$ or larger and $n \geq 2 k+1 \geq 5$. Then

$$
e(G) \leq \max \left\{h_{1}(n, k), h_{2}(n, k)\right\}
$$

and this bound is the best possible. Moreover, only the graphs $H_{1}(n, k)$ and $H_{2}(n, k)$ could be extremal. For further explanation and background see the recent survey [8].

The other result we need is due to Brandt [4]: Let $G$ be a non-bipartite graph of order $n$ and suppose that

$$
e(G)>(n-1)^{2} / 4+1
$$

then $G$ contains cycles of every length between 3 and the length of its longest cycle.

Proof of Lemma 2; The inequality $e(G) \leq \operatorname{ex}\left(n, C_{2 k+1}\right)$ follows from the definition. Suppose that here equality holds. Apply Brandt's theorem (44). We obtain that $G$ contains cycles of all lengths $3,4, \ldots, \ell$ where $\ell$ stands for the longest cycle length in $G$. It follows that $\ell \leq 2 k$. Kopylov's theorem (3) implies that

$$
\max \left\{g(n, k),\left\lfloor n^{2} / 4\right\rfloor\right\} \leq \operatorname{ex}\left(n, C_{2 k+1}\right)=e(G) \leq \max \left\{h_{1}(n, k), h_{2}(n, k)\right\} .
$$

Since $g(n, k)>h_{2}(n, k)$ except for $(n, k) \in\{(5,2),(6,2)\}$ and $g(n, k)>h_{1}(n, k)$ except if $n$ is in the form $n=(s-1)(2 k-1)+r$ where $s \geq 2$ and $r \in\{k, k+1\}$ we obtain that $e(G)=h_{1}(n, k), n$ should be in this form, and $G=H_{1}(n, k)$.

Finally, $h_{1}(n, k)<\left\lfloor n^{2} / 4\right\rfloor$ for $n \geq 4 k$ so we obtain that indeed $n \in\{3 k-1,3 k\}$. 


\section{The proof of Theorem 1}

Suppose that $G$ is an extremal $C_{2 k+1}$-free graph, $e(G)=\operatorname{ex}\left(n, C_{2 k+1}\right)$. Then $G$ is connected. Consider the cactus-like block-decomposition of $G, V(G)=V_{1} \cup V_{2} \cup$ $\cdots \cup V_{s}$, where the induced subgraphs $G\left[V_{i}\right]$ are either edges or maximal 2-connected subgraphs of $G$. Let $n_{i}:=\left|V_{i}\right|$, we have $n-1=\sum_{i}\left(n_{i}-1\right)$, and each $n_{i} \geq 2$. We have $e\left(G\left[V_{i}\right]\right)=\operatorname{ex}\left(n_{i}, C_{2 k+1}\right)$ otherwise one can replace $G\left[V_{i}\right]$ by an extremal graph of the same order $n_{i}$ and obtain another $C_{2 k+1}$-free graph of size larger than $e(G)$. Therefore $e\left(G\left[V_{i}\right]\right) \geq\left\lfloor n_{i}^{2} / 4\right\rfloor$ and there are three types of blocks

- complete graphs (if $n_{i} \leq 2 k$ ),

- bipartite blocks with $e\left(G\left[V_{i}\right]\right)=\left\lfloor n_{i}^{2} / 4\right\rfloor$. Finally,

- if $n_{i} \geq 2 k+1$ and $G\left[V_{i}\right]$ is not bipartite then Lemma2 2 implies that $n_{i} \in\{3 k-1,3 k\}$ and $G\left[V_{i}\right]=H_{1}\left(n_{i}, k\right)$.

We may rearrange the graphs $G\left[V_{i}\right]$ and the sets $V_{i}$ such a way that they share a common vertex $v \in \cap V_{i}$ and otherwise the sets $V_{i} \backslash\{v\}$ are pairwise disjoint. The obtained new graph $G^{*}$ also $C_{2 k+1}$-free and extremal, it has the same size and order as $G$ has.

If $s=1$ then we are done. Suppose $s \geq 2$. If all blocks are complete graphs, then $e(G) \leq g(n, k)$. Since $g(n, k)<e\left(T_{n, 2}\right)$ for $n>4 k-1$ we get that $n \leq 4 k-1$ and $G^{*}$ (and $G$ ) has only two blocks and at least one of them is of size $2 k$.

Finally, suppose that there are two blocks $V_{i}$ and $V_{j},\left|V_{i}\right|=a$ and $\left|V_{j}\right|=b$, such that $G\left[V_{i}\right]$ and $G\left[V_{j}\right]$ are not both complete subgraphs. We claim that in this case one can remove the edges of $G\left[V_{i}\right]$ and $G\left[V_{j}\right]$ from $G^{*}$ and place a copy of $T_{a+b-1,2}$ or some other graph $L$ onto $V_{i} \cup V_{j}$ such that the obtained new graph is $C_{2 k+1}$-free and it has more edges than $e(G)$, a contradiction.

Indeed, if $G\left[V_{i}\right]$ is a large bipartite graph, $a:=n_{i} \geq 2 k+1, G\left[V_{i}\right]=T_{a, 2}$ and $G\left[V_{j}\right]$ is a complete bipartite graph, too, then we can increase $e\left(G^{*}\right)$ since

$$
e\left(T_{a, 2}\right)+e\left(T_{b, 2}\right) \leq \frac{1}{4} a^{2}+\frac{1}{4} b^{2}<\left\lfloor\frac{1}{4}(a+b-1)^{2}\right\rfloor=e\left(T_{a+b-1,2}\right) .
$$

In the remaining cases the inequalities concerning the number of edges of $e(L)$ are just elementary high school algebra. If $G\left[V_{i}\right]=T_{a, 2}$ and $G\left[V_{j}\right]=H_{1}(b, k)$ or $K_{b}$ then we can replace them again by a complete bipartite graph $T_{a+b-1,2}$. From now on, we may suppose that each block is either a complete graph (of size at most $2 k$ ) or an $H_{1}(a, k)$. If $G\left[V_{i}\right]=H_{1}(a, k)$ for some $a \in\{3 k-1,3 k\}$ and $G\left[V_{j}\right]=H_{1}(b, k)$ (with $b \in\{3 k-1,3 k\})$ or $G\left[V_{j}\right]=K_{b}$ with $k \leq b \leq 2 k$ then we replace $G\left[V_{i}\right] \cup G\left[V_{j}\right]$ again by a $T_{a+b-1,2}$. Finally, if $G\left[V_{i}\right]=H_{1}(a, k)$ for some $a \in\{3 k-1,3 k\}$ and $G\left[V_{j}\right]=K_{b}$ with $2 \leq b \leq k$ then we replace $G\left[V_{i}\right] \cup G\left[V_{j}\right]$ by two complete graphs of sizes $2 k$ 
Füredi-Gunderson: Extremal numbers for odd cycles

and $a+b-2 k$ and use $e\left(H_{1}(a, k)\right)+e\left(K_{b}\right)<e(B(a+b-1 ; 2 k, a+b-2 k))$ to get a contradiction. This completes the proof of the claim and Theorem 1 .

\section{References}

[1] B. Bollobás, Extremal Graph Theory, Academic Press, New York, 1979. 1, 2

[2] J. A. Bondy, Pancyclic graphs I, J. Combin. Theory Ser. B 11 (1971), 80-84. 2

[3] J. A. Bondy, Large cycles in graphs, Discrete Math. 1 (1971), 121-132. 1, 2

[4] S. Brandt, A sufficient condition for all short cycles, in: 4th Twente Workshop on Graphs and Combinatorial Optimization (Enschede, 1995). Discrete Appl. Math. 79

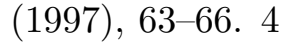

[5] T. Dzido, A note on Turán numbers for even wheels, Graphs Combin. 29 (2013), 1305-1309. 2

[6] P. Erdős and T. Gallai, On maximal paths and circuits of graphs, Acta Math. Acad. Sci. Hung. 10 (1959), 337-356. 2

[7] R. J. Faudree and R. H. Schelp, Path Ramsey numbers in multicolorings, J. Combin. Theory Ser. B 19 (1975), 150-160. 2

[8] Z. Füredi and M. Simonovits, The history of degenerate (bipartite) extremal graph problems, Bolyai Soc. Studies (The Erdős Centennial) 25 (2013), 167-262. 4

[9] G. N. Kopylov, Maximal paths and cycles in a graph, Dokl. Akad. Nauk SSSR 234 (1977), no. 1, 19-21. (English translation: Soviet Math. Dokl. 18 (1977), no. 3, 593596.) 2, 4

[10] W. Mantel, Solution to Problem 28, by H. Gouwentak, W. Mantel, J. Teixeira de Mattes, F. Schuh, and W. A. Wythoff, Wiskundige Opgaven 10 (1907), 60-61. 2

[11] M. Simonovits, Extremal graph problems with symmetrical extremal graphs, additional chromatic conditions, Discrete Math. 7 (1974), 349-376. 2

[12] P. Turán, Eine Extremalaufgave aus der Graphentheorie (in Hungarian), Math. Fiz Lapook 48 (1941), 436-452. 2

[13] P. Turán, On the theory of graphs, Colloq. Math. 3 (1954), 19-30. 2

[14] D. R. Woodall, Sufficient conditions for circuits in graphs, Proc. London Math. Soc. (3) 24 (1972), 739-755. 1, 2

[15] D. R. Woodall, Maximal circuits of graphs I, Acta Math. Acad. Sci. Hungar. 28 (1976), 77-80. 2 